\title{
The Reliability of a System Involving Change Points
}

\author{
Amos E. Gera \\ SCE College of Engineering, Zabotinsky 84, Ashdod, Israel. \\ Corresponding author: amosgera@gmail.com
}

(Received May 15, 2020; Accepted August 7, 2020)

\begin{abstract}
The reliability of a system having some change points is presented. The technique of calculation is based on a previously developed TFCF procedure for evaluating the reliability for i.i.d. component. It involves the use of some auxiliary functions to set up a set of recursive relations. The resultant equations are solved numerically. An extension to the more general TSCSTFCF procedure and its application to start-up demonstration tests is given. Also, in case of testing, the possibility of carrying out simultaneous tests on a set of units is considered.
\end{abstract}

Keywords- Reliability, Change points, Start-up demonstration tests, Point mass distribution.

$\begin{array}{ll}\begin{array}{ll}\text { Notation } \\ \text { TSCSTFCF }\end{array} & \text { total successes consecutive successes total failures consecutive failures } \\ k_{s} & \text { total number of successes } \\ k_{f} & \text { total number of failures } \\ k_{c s} & \text { length of run of successes } \\ k_{c f} & \text { length of run of failures } \\ T_{n, s} & \text { the total number of successes up to the } n^{\prime} \text { th test } \\ T_{n, f} & \text { the total number of failures up to the } n \text { 'th test } \\ L_{n, s} & \text { the length of the largest run of successes up to } n \\ X_{n} & \text { the output at the } n \text {-th test: }=1 \text { success, }=0 \text { failure } \\ N & \text { the random number of tests till stopping } \\ \mathrm{u}[.] & \text { the unit step function } \\ \delta[.] & \text { the delta function. }\end{array}$

\section{Introduction}

The way of evaluating the reliability of systems consisting of $\mathrm{n}$ identical components is commonly known. An example of a reference book on the basic theory of consecutive k-out-of-n systems is of Kuo and Zou (2003). A comparison of various procedures for performing tests that are based on this theory has been presented by Gera (2018). Matters become more cumbersome when we must deal with systems that have various parts with different reliabilities. Some work has been carried out for systems with non-identical elements (see for instance Smith and Griffith, 2011). The application of the theory to start-up demonstration tests has been presented by Balakrishnan et al. (2014) and others.

We divide the system into several parts (at so-called "change points") so that the components within each part are identical from the point of view of their reliabilities. Eryilmaz (2016) handled the case of a single change point. An extension to the case of two change points has been presented by Peng and Xiao (2018). They developed non-recursive formulas for calculating the survival function and for the reliability of such systems. An example of a pipeline which transports oil from one location to the other has been shown. Due to different environmental 
International Journal of Mathematical, Engineering and Management Sciences

Vol. 6, No. 1, 296-308, 2021

https://doi.org/10.33889/IJMEMS.2021.6.1.019

conditions along the various parts of the pipeline, there are assumed different reliabilities for each of its parts.

A general method for analyzing the survival function and the point mass distribution function for systems having components (or tests) with identical and independent reliabilities (probabilities of success) has been introduced by Gera $(2010,2011,2019)$ - the TSCSTFCF procedure. It has been used for start-up demonstration tests. The procedure is based on some auxiliary functions which lead to a set of recursive relations that are normally solved numerically. This method is extended here to include change points.

\section{A Single Change Point}

A change point is given at $n=n_{1}$ within the distribution of the probabilities of success throughout the different tests:

$$
p\left(n^{\prime}\right)=\left\{\begin{array}{cc}
p_{1} & 1 \leq n^{\prime} \leq n_{1} \\
p_{2} & n_{1}<n^{\prime}
\end{array}\right\}
$$

A consecutive $\mathrm{k}_{\mathrm{cs}}$-out-of-n $\mathrm{G}$ :system is considered.

Let $L_{n, s}$ be the length of the largest run of successes up to $n$ and let $T_{n, s}$ be the total number of successes up to the $n . X_{n}$ is the output at the n'th test:

$$
X_{n}=\left\{\begin{array}{lcc}
1 & \text { success at } n \\
0 & \text { failue at } n
\end{array}\right\}
$$

Define the auxiliary functions for $\mathrm{r}=0,1$ :

$$
f_{r}(i, n)=P\left\{T_{n s}=i, L_{n s}<k_{c s}, X_{n}=r\right\}
$$

Gera $(2011,2019)$ presented interconnecting relations for a more general case (TSCSTFCF). Meanwhile we confine the presentation to the CS (consecutive successes) procedure.

For every i,n

$$
f_{0}(i, n)=\left\{\begin{array}{ll}
q_{1} \cdot\left[f_{0}(i, n-1)+f_{1}(i,, n-1)\right] & n \leq n_{1} \\
q_{2} \cdot\left[f_{0}(i, n-1)+f_{1}(i, n-1)\right] & n>n_{1}
\end{array}\right\}
$$

Also,

$$
\begin{aligned}
& n \leq n_{1}: \\
& f_{1}(i, n)=\sum_{a=1}^{\min \left(k_{c s}-1, i, n_{1}\right)} p_{1}{ }^{a} \cdot f_{0}(i-a, n-a)
\end{aligned}
$$

where,

$f_{l}(0,0)=1$ 
International Journal of Mathematical, Engineering and Management Sciences

Vol. 6, No. 1, 296-308, 2021

https://doi.org/10.33889/IJMEMS.2021.6.1.019

and

$$
\begin{aligned}
& n>n_{1}: \\
& \left.f_{1}(i, n)=\sum_{a=1}^{\min \left(n-n_{1}, k_{c s}-1, i\right)} p_{2}{ }^{a} \cdot f_{0}(i-a, n-a)+\sum_{b=1}^{\min \left(k_{c s}-1-\left(n-n_{1}\right), i, n-n_{1}\right)} p_{2}{ }^{n-n_{1}} \cdot p_{1}{ }^{b} \cdot f_{0}\left(i-b-\left(n-n_{1}\right), n-b-\left(n-n_{1}\right)\right)\right) \\
& \\
& \quad+p_{2}{ }^{n-n_{1}} \cdot p_{1}{ }^{n_{1}} \cdot \delta[i-n] \cdot u\left[k_{c s}-1-i\right]
\end{aligned}
$$

where, $\delta[$.$] is the delta function and \mathrm{u}[$.$] is the unit step function.$

The boundary conditions for the system of equations (4)-(6) are:

$$
\begin{aligned}
& f_{0}(0, n)=\left\{\begin{array}{cc}
q_{1}{ }^{n} & n \leq n_{1} \\
q_{1}{ }^{n_{1}} q_{2}{ }^{n-n_{1}} & n>n_{1}
\end{array}\right\} \\
& f_{1}(0, n)=0 \\
& f_{0}(1,1)=0 \\
& f_{1}(1, n)=\left\{\begin{array}{cc}
p_{1} q_{1}{ }^{n-1} & n \leq n_{1} \\
p_{2} q_{1}{ }^{{ }_{1}} q_{2}{ }^{n-n_{1}-1} & n>n_{1}
\end{array}\right\}
\end{aligned}
$$

Thereafter,

$$
P\{N>n\}=\sum_{i=0}^{n}\left\{f_{0}(i, n)+f_{1}(i, n)\right\}
$$

\begin{tabular}{|c|c|c|c|c|c|c|c|}
\hline $\mathrm{N}$ & 1 & 2 & 3 & 4 & 5 & 6 & 7 \\
\hline $\mathrm{P}\{\mathrm{N}>\mathrm{n}\}$ & 1 & 1 & 0.5781 & 0.5078 & 0.4609 & 0.4297 & 0.3936 \\
\hline $\mathrm{P}\{\mathrm{N}=\mathrm{n}\}$ & 0 & 0 & $\begin{array}{c}0.4219 \\
\text { 'sss' }\end{array}$ & $\begin{array}{c}0.0703 \\
\text { 'fsss' }\end{array}$ & $\begin{array}{l}0.0469 \\
\text { '1fsss' }\end{array}$ & $\begin{array}{l}0.0313 \\
\text { '11fsss' }\end{array}$ & $\frac{0.0361}{s s s} f s s s^{\prime}$ \\
\hline $\mathrm{R}^{(\mathrm{G})}(\mathrm{n})$ & 0 & 0 & 0.4219 & 0.4922 & 0.5391 & 0.5703 & 0.6064 \\
\hline
\end{tabular}

and the reliability of a $\mathrm{G}$ : system up to $\mathrm{n}$ is given by

$$
R^{(G)}(n)=1-P\{N>n\}
$$

Example A: Calculation of $\mathrm{R}^{(\mathrm{G})}(\mathrm{n})$ for $\mathrm{p}_{1}=0.75, \mathrm{p}_{2}=0.5$; change point at $\mathrm{n}_{1}=3, \mathrm{k}_{\mathrm{cs}}=3$. 's' stands for success and 'f' stands for failure, '1' may be any of them (Table 1).

Table 1. $\mathrm{R}^{(\mathrm{G})}(\mathrm{n})$ for $\mathrm{n}_{1}=3, \mathrm{k}_{\mathrm{cs}}=3, \mathrm{p}_{1}=0.75, \mathrm{p}_{2}=0.5$.

The above equality (9) may be modified to include also the possibility of having a total number of successes $\left(\mathrm{k}_{\mathrm{s}}\right)$ till $\mathrm{n}$. This is the total successes consecutive successes procedure (TSCS).

Example B: Like example A with the addition of $\mathrm{k}_{\mathrm{s}}=4$ (Table 2). 
International Journal of Mathematical, Engineering and Management Sciences

Vol. 6, No. 1, 296-308, 2021

https://doi.org/10.33889/IJMEMS.2021.6.1.019

Table 2. $\mathrm{R}^{(\mathrm{G})}(\mathrm{n})$ for $\mathrm{n}_{1}=3, \mathrm{k}_{\mathrm{cs}}=3, \mathrm{p}_{1}=0.75, \mathrm{p}_{2}=0.5, \mathrm{k}_{\mathrm{s}}=4$.

\begin{tabular}{|l|c|c|c|c|c|c|c|}
\hline $\mathrm{n}$ & 1 & 2 & 3 & 4 & 5 & 6 & 7 \\
\hline $\mathrm{P}\{\mathrm{N}>\mathrm{n}\}$ & 1 & 1 & 0.5781 & 0.5078 & 0.4258 & 0.3242 & 0.2295 \\
\hline $\mathrm{P}\{\mathrm{N}=\mathrm{n}\}$ & 0 & 0 & 0.4219 & 0.0703 & 0.0820 & 0.1016 & 0.0947 \\
& & & $\begin{array}{c}\text { 'sss' } \\
\text { 'fsss' }\end{array}$ & $\begin{array}{c}\text { '1fsss', 'ssfss' } \\
\text { 'sffss', 'fsfsss' }\end{array}$ & $\begin{array}{c}\text { 'fffsss','ssffss } \\
\text { 'sfsfss','fssfss' } \\
\text { 'ssfsfs','sfssfs' }\end{array}$ \\
\hline $\mathrm{R}^{(\mathrm{G})}(\mathrm{n})$ & 0 & 0 & 0.4219 & 0.4922 & 0.5747 & 0.6758 & 0.7705 \\
\hline
\end{tabular}

Obviously, the TSCS procedure exhibits increased values of the reliability compared to those of the CS system.

\section{Two Change Points}

A generalization to two change points at $\mathrm{n}_{1}, \mathrm{n}_{2}$ is presented. Accordingly,

$$
p(n)=\left\{\begin{array}{cc}
p_{1} & 1 \leq n \leq n_{1} \\
p_{2} & n_{1}<n \leq n_{1}+n_{2} \\
p_{3} & n_{1}+n_{2}<n
\end{array}\right\}
$$

The auxiliary functions (3) are as before for the CS procedure. The modified interconnecting relations are:

For $n \leq n_{1}$ :

$f_{1}(i, n)=\sum_{a=1}^{\min \left(k_{c s}-1, i, n_{1}\right)} p_{1}{ }^{a} \cdot f_{0}(i-a, n-a)$

where, $f_{0}(0,0)=1$.

For $n_{1}<n \leq n_{1}+n_{2}$ :

$$
\begin{aligned}
f_{1}(i, n)= & \sum_{a=1}^{\min \left(n-n_{1}, k_{c s}-1, i\right)} p_{2}^{a} \cdot f_{0}(i-a, n-a) \\
& +\sum_{b=n-n_{1}+1}^{\min \left(k_{c s}-1-\left(n-n_{1}\right), i, n-1\right)} p_{2}{ }^{n-n_{1}} \cdot p_{1}{ }^{b} \cdot f_{0}\left(i-b-\left(n-n_{1}\right), n-b-\left(n-n_{1}\right)\right) \\
& +p_{2}{ }^{n-n_{1}} p_{1}{ }^{n_{1}} \cdot \delta[i-n] \cdot u\left[k_{c s}-1-i\right]
\end{aligned}
$$


International Journal of Mathematical, Engineering and Management Sciences

Vol. 6, No. 1, 296-308, 2021

https://doi.org/10.33889/IJMEMS.2021.6.1.019

$$
\begin{aligned}
\text { For } n> & \left(n_{1}+n_{2}\right): \\
f_{1}(i, n)= & \sum_{a=1}^{\min \left[n-\left(n_{1}+n_{2}\right), i, k_{c s}-1\right]} p_{3}{ }^{a} \cdot f_{0}(i-a, n-a) \\
& +\sum_{b=1}^{\min \left(n-n_{1}, k_{c s}-1-\left[n-\left(n_{1}+n_{2}, i\right)\right]\right)} p_{3}{ }^{n-\left(n_{1}+n_{2}\right)} p_{2}{ }^{b} \cdot f_{0}\left(i-b-\left[n-\left(n_{1}+n_{2}\right), n-b-\left[n-\left(n_{1}+n_{2}\right)\right]\right]\right) \\
& +\sum_{c=1}^{\min \left(n-1, k_{c s}-1-\left(n-n_{1}\right)\right)} p_{3}{ }^{n-\left(n_{1}+n_{2}\right)} p_{2}{ }^{{ }_{2}} p_{1}{ }^{c} \cdot f_{0}\left(i-c-\left(n-n_{1}\right), n-c-\left(n-n_{1}\right)\right) \\
& +p_{3}{ }^{n-\left(n_{1}+n_{2}\right)} p_{2}{ }^{n_{2}} p_{1}{ }^{n_{1}} \cdot \delta[i-n] \cdot u\left[k_{c s}-1-i\right]
\end{aligned}
$$

For $n>1$ :

$$
f_{0}(i, n)=\left\{\begin{array}{lc}
q_{1}\left[f_{0}(i, n-1)+f_{1}(i, n-1)\right] & 1 \leq n \leq n_{1} \\
q_{2}\left[f_{0}(i, n-1)+f_{1}(i, n-1)\right] & n_{1}<n \leq n_{1}+n_{2} \\
q_{3}\left[f_{0}(i, n-1)+f_{1}(i, n-1)\right] & n_{1}+n_{2}<n
\end{array}\right\}
$$

The boundary conditions for the system of equations (12)-(15) are:

$$
f_{0}(1,1)=0
$$

$$
\begin{gathered}
f_{0}(0, n)=\left\{\begin{array}{cc}
q_{1}{ }^{n} & n \leq n_{1} \\
q_{1}{ }^{n_{1}} q_{2}{ }^{n-n_{1}} & n_{1}+n_{2} \geq n>n_{1} \\
q_{1}{ }^{{ }^{n}} q_{2}{ }^{{ }_{2}} q_{3}{ }^{n-\left(n_{1}+n_{2}\right)} & n>n_{1}+n_{2}
\end{array}\right\} \\
f_{1}(1, n)=\left\{\begin{array}{cc}
p_{1} q_{1}{ }^{n-1} & n \leq n_{1} \\
p_{2} q_{1}{ }^{{ }_{1}} q_{2}{ }^{n-n_{1}-l_{1}} & n_{1}+n_{2} \geq n>n_{1} \\
p_{3} q_{1}{ }^{{ }_{1}} q_{2}{ }^{n_{2}} q_{3}{ }^{n-\left(n_{1}+n_{2}\right)-1} & n>n_{1}+n_{2}
\end{array}\right\}
\end{gathered}
$$

It may be noted that for a CF system (F: system), the reliability is given by:

$$
R^{(F)}(n)=1-\sum_{n^{\prime}=1}^{n} P\left\{N=n^{\prime}\right\}
$$

The auxiliary functions are evaluated as before but with the proper interchanging of the roles of the $\mathrm{p}_{\mathrm{r}}$ and $\mathrm{q}_{\mathrm{r}}$ variables.

Example C: The reliability of an oil pipeline system. It is considered as an F: system composed of three parts. Considering various combinations of the parameters of the system, we get identical results to those of Peng and Xiao (2018) as presented in their Table 1.

Example D: CF system, $\mathrm{k}_{\mathrm{cf}}=3, \mathrm{n}_{1}=5, \mathrm{n}_{2}=9, \mathrm{n}_{3}=6$. The reliability is evaluated at $\mathrm{n}=20$ (Figure 1 ). 
International Journal of Mathematical, Engineering and Management Sciences

Vol. 6, No. 1, 296-308, 2021

https://doi.org/10.33889/IJMEMS.2021.6.1.019

$\mathrm{R} 1$ is the reliability due to $\mathrm{p}_{1}(\mathrm{x})=0.88-\mathrm{x}, \mathrm{p}_{2}=0.92, \mathrm{p}_{3}=0.95$

$\mathrm{R} 2$ is the reliability due to $\mathrm{p}_{1}=0.88, \mathrm{p}_{2}(\mathrm{x})=0.92-\mathrm{x}, \mathrm{p}_{3}=0.95$

$\mathrm{R} 3$ is the reliability due to $\mathrm{p}_{1}=0.88, \mathrm{p}_{2}=0.92 . \mathrm{p}_{3}(\mathrm{x})=0.95-\mathrm{x}$.

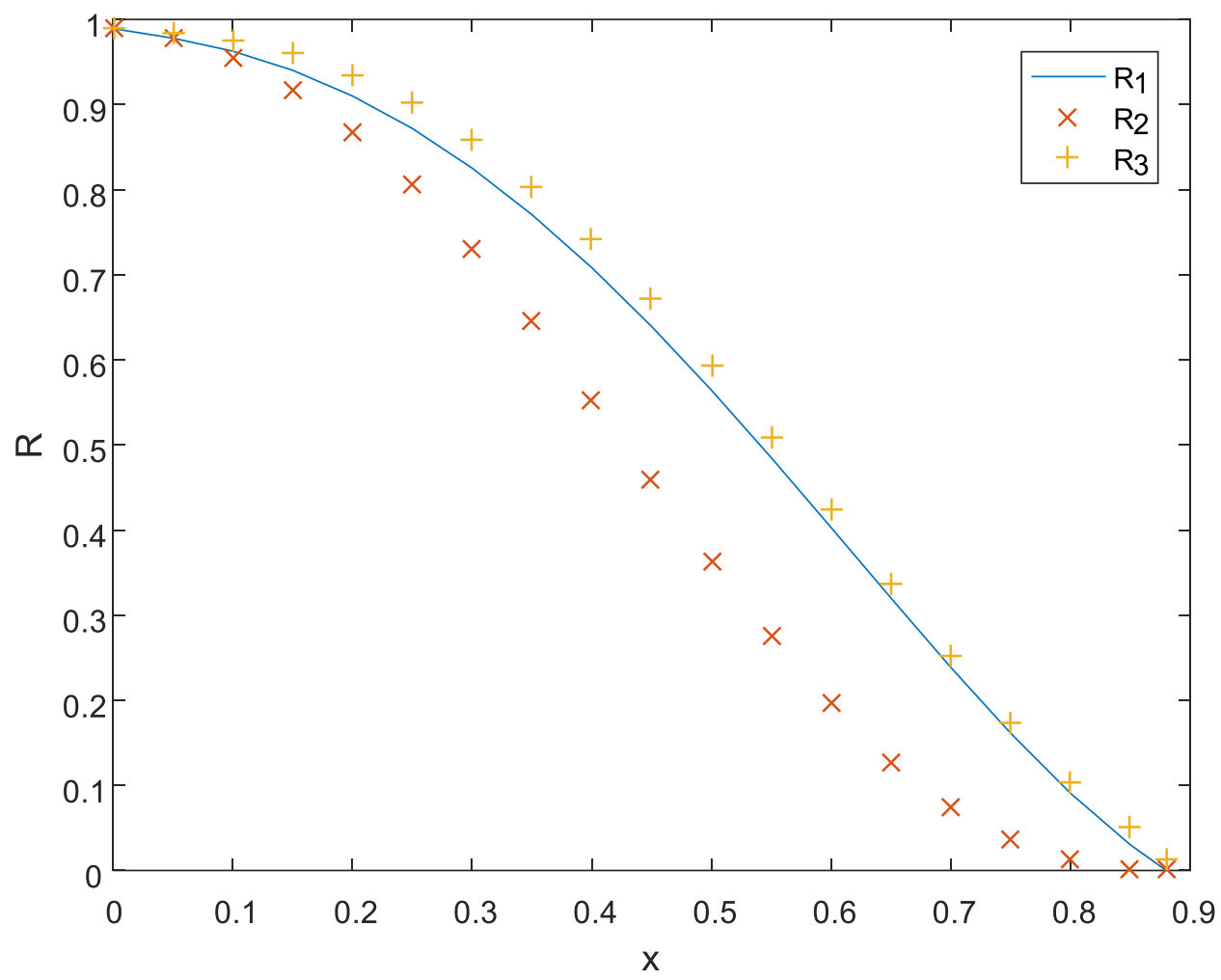

Figure 1. The change in the value of $R(20)$ due to changes in $\mathrm{p}_{1}, \mathrm{p}_{2}, \mathrm{p}_{3}, \mathrm{k}_{\mathrm{cf}}=3, \mathrm{n}_{1}=5, \mathrm{n}_{2}=9, \mathrm{n}_{3}=6$.

\section{Extension of the Procedure}

Like for the case of a single change point, we may generalize the above procedure to include also the total number of failures (the TFCF procedure).

Let $\mathrm{k}_{\mathrm{f}}$ denote the total number of failures. The tail distribution of $\mathrm{N}$ is given in this case by:

$$
P\{N>n\}=\sum_{i=\max \left(0, n-k_{f}+1\right)}^{\min \left(k_{s}-1, n\right)}\left\{f_{0}(i, n)+f_{1}(i, n)\right\}
$$

Example D: The reliability of a TFCF system, $\mathrm{n}=20$, various values of $\mathrm{k}_{\mathrm{f}}, \mathrm{k}_{\mathrm{cf}}$ (Table 3 ). 
International Journal of Mathematical, Engineering and Management Sciences

Vol. 6, No. 1, 296-308, 2021

https://doi.org/10.33889/IJMEMS.2021.6.1.019

Table 3. $\mathrm{R}^{(\mathrm{F})}(20)$, various values of $\mathrm{n}_{1}, \mathrm{n}_{2}, \mathrm{k}_{\mathrm{f}}, \mathrm{k}_{\mathrm{cf}}$.

\begin{tabular}{|c|c|c|c|c|c|c|c|c|}
\hline $\mathrm{k}_{\mathrm{f}}$ & $\mathrm{k}_{\mathrm{cf}}$ & $\mathrm{n}_{1}$ & $\mathrm{n}_{2}$ & $\mathrm{n}_{3}$ & $\mathrm{p}_{1}$ & $\mathrm{p}_{2}$ & $\mathrm{p}_{3}$ & $\mathrm{R}(20)$ \\
\hline- & 3 & 5 & 9 & 6 & 0.88 & 0.92 & 0.95 & 0.9893 \\
\hline 5 & - & $"$ & $"$ & $"$ & 0.88 & 0.92 & 0.95 & 0.9813 \\
\hline 5 & 3 & $"$ & $"$ & " & 0.88 & 0.92 & 0.95 & 0.9744 \\
\hline- & 3 & $"$ & $"$ & $"$ & 0.92 & 0.92 & 0.92 & 0.9915 \\
\hline 5 & - & $"$ & $"$ & $"$ & 0.92 & 0.92 & 0.92 & 0.9817 \\
\hline 5 & 3 & $"$ & $"$ & $"$ & 0.92 & 0.92 & 0.92 & 0.9743 \\
\hline- & 3 & 6 & 8 & 6 & 0.88 & 0.92 & 0.95 & 0.9883 \\
\hline 5 & - & $"$ & $"$ & $"$ & 0.88 & 0.92 & 0.95 & 0.9794 \\
\hline 5 & 3 & $"$ & $"$ & " & 0.88 & 0.92 & 0.95 & 0.9719 \\
\hline- & 4 & 5 & 9 & 6 & 0.88 & 0.92 & 0.95 & 0.9991 \\
\hline 6 & - & $"$ & $"$ & $"$ & 0.88 & 0.92 & 0.95 & 0.9962 \\
\hline 6 & 4 & $"$ & $"$ & $"$ & 0.88 & 0.92 & 0.95 & 0.9955 \\
\hline
\end{tabular}

\section{Application to Start-Up Demonstration Tests}

Start-up demonstration sets of tests are often assumed to be i.i.d.(identical independent). In contrast, the probabilities of success of each test may change due to different environmental conditions. Thus, we may apply the above theory also to such tests. Here we may apply a generalized version of the former CSCF, TSCS and TFCF procedures - the TSCSTFCF procedure, Gera $(2011,2018)$. The set of tests is stopped if either there is a total number of successes or a run of consecutive successes or if we observe a certain total number of failures or a run of failures. Accordingly, a decision regarding the acceptance or rejection of the tested unit is then taken. The aim here is to shorten as much as possible the number of tests subject to some constraints.

Referring to (19), the point distribution of $\mathrm{N}$ will be given by (for $\mathrm{n}>1$ ):

$$
P\{N=n\}=P\{N>n-1\}-P\{N>n\}
$$

The generalization of the auxiliary functions and the interconnecting relations between them has already been given before for the i.i.d. case.

$n>i \geq 1$ :

$$
f_{0}(i, n)=\left\{\begin{array}{cc}
\sum_{a_{1}=1}^{\min \left(k_{c f}-1, n\right)} q_{1}^{a_{1}} \cdot f_{1}\left(i, n-a_{1}\right) & n \leq n_{1} \\
\sum_{a_{2}=1}^{\min \left(n_{1}, k_{c f}-1-\left(n-n_{1}\right)\right)} q_{2}{ }^{\left.a_{2}-1, n-n_{1}\right)} \cdot f_{1}\left(i, n-a_{2}\right)+\sum_{a_{1}=1}^{n q_{2}{ }^{n-n_{1}}} q_{1}{ }^{a_{1}} \cdot f_{1}\left(i, n_{1}-a_{1}\right) & n>n_{1}
\end{array}\right\}
$$

where $f_{1}(0,0)=1$ 
International Journal of Mathematical, Engineering and Management Sciences

Vol. 6, No. 1, 296-308, 2021

https://doi.org/10.33889/IJMEMS.2021.6.1.019

$n>i \geq 1$ :

$f_{1}(i, n)=\left\{\begin{array}{cc}\sum_{b_{1}=1}^{\min \left(k_{c s}-1, n\right)} p_{1}^{b_{1}} \cdot f_{0}\left(i-b_{1}, n-b_{1}\right) & n \leq n_{1} \\ \sum_{b_{2}=1}^{\min \left(k_{c s}-1, n-n_{1}\right)} p_{2}^{b_{2}} \cdot f_{0}\left(i-b_{2}, n-b_{2}\right)+\sum_{b_{1}=1}^{\min \left(n_{1}, k_{c s}-1-\left(n-n_{1}\right)\right)} p_{2}^{n-n_{1}} p_{1}^{b_{1}} \cdot f_{0}\left(i-b_{1}-\left(n-n_{1}\right), n_{1}-b_{1}\right) & n>n_{1}\end{array}\right\}$

where $f_{0}(0,0)=1$

Like before, appropriate boundary conditions must be taken into account. Then, use is made of (19) and (20).

Example E: CS procedure. The point mass distribution for some values of $n$. $p_{1}=0.75, p_{2}=0.5$, $\mathrm{n}_{1}=2, \mathrm{k}_{\mathrm{cs}}=2$ (Table 4$)$.

Table 4. $\mathrm{P}\{\mathrm{N}=\mathrm{n}\}$ for $. \mathrm{p}_{1}=0.75, \mathrm{p}_{2}=0.5, \mathrm{n}_{1}=2, \mathrm{k}_{\mathrm{cs}}=2$.

\begin{tabular}{|l|c|c|c|c|c|}
\hline $\mathrm{n}$ & 1 & 2 & 3 & 4 & 5 \\
\hline $\mathrm{P}\{\mathrm{N}=\mathrm{n}\}$ & 0 & 0.5625 & 0.0938 & 0.0625 & 0.0273 \\
& & 'ss' & 'fss' & 'ffss' & '( (ss)fss' \\
\hline
\end{tabular}

Example F: TSCSTFCF procedure. $\mathrm{p}_{1}=0.75, \mathrm{p}_{2}=0.5, \mathrm{n}_{1}=3, \mathrm{k}_{\mathrm{cs}}=\mathrm{k}_{\mathrm{cf}}=2$, without $(1)$ and with the addition of the constraint $\mathrm{k}_{\mathrm{s}}=\mathrm{k}_{\mathrm{f}}=3$ (2) (Table 5).

Table 5. $\mathrm{P}\{\mathrm{N}=\mathrm{n}\}$ for $\mathrm{p}_{1}=0.75, \mathrm{p}_{2}=0.5$ : $\operatorname{CSCF}\left(\mathrm{k}_{\mathrm{cs}}=\mathrm{k}_{\mathrm{cf}}=2\right)(1)$, TSCSTFCF $\left(\mathrm{k}_{\mathrm{cs}}=\mathrm{k}_{\mathrm{cf}}=2, \mathrm{k}_{\mathrm{s}}=\mathrm{k}_{\mathrm{f}}=3\right)(2)$.

\begin{tabular}{|l|c|c|c|c|c|c|c|}
\hline $\mathrm{n}$ & 1 & 2 & 3 & 4 & 5 & 6 & 7 \\
\hline $\begin{array}{l}\mathrm{P}\{\mathrm{N}=\mathrm{n}\} \\
(1)\end{array}$ & 0 & $\begin{array}{c}0.625 \\
\text { 'ss','ff' }\end{array}$ & $\begin{array}{c}0.1875 \\
\text { 'sff','fss' }\end{array}$ & $\begin{array}{c}0.0938 \\
\text { 'fsff','sfss' }\end{array}$ & 0.0469 & 0.0234 & 0.0117 \\
\hline $\begin{array}{l}\mathrm{P}\{\mathrm{N}=\mathrm{n}\} \\
(2)\end{array}$ & 0 & 0.625 & 0.1875 & 0.0938 & 0.0938 & 0 & 0 \\
\hline
\end{tabular}

Considering two change points, we derive the following generalization of the previous interconnecting relations:

For $\mathrm{n}>\mathrm{i} \geq 1$ :

$$
f_{0}(i, n)=\left\{\begin{array}{cc}
\sum_{a_{1}=1}^{\min \left(k_{c f}-1, n\right)} q_{1}^{a_{1}} \cdot f_{1}\left(i, n-a_{1}\right) & n \leq n_{1} \\
\sum_{a_{2}=1}^{\min \left(k_{c f}-1, n-n_{1}\right)} q_{2}^{a_{2}} \cdot f_{1}\left(i, n-a_{2}\right)+\sum_{a_{3}=1}^{\min \left(n_{1}, k_{c f}-1-\left(n-n_{1}\right)\right)} q_{2}{ }^{n-n_{1}} q_{1}{ }^{a_{1}} \cdot f_{1}\left(i, n_{1}-a_{1}\right) & \left.n_{1}<n \leq\left(n_{1}+n_{2}\right)\right\} \\
\sum_{a_{3}\left(k_{c f}-1, n-\left(n_{1}+n_{2}\right)\right)} q^{a_{3}} \cdot f_{1}\left(i, n-a_{3}\right)+\sum_{a_{2}=1}^{\min \left(k_{c f}-1-\left[n-\left(n_{1}+n_{2}\right)\right], n_{2}\right)} q^{n-\left(n_{1}+n_{2}\right)} q_{2}{ }^{a_{2}} \cdot f_{1}\left(i, n_{1}+n_{2}-a_{2}\right)+S_{3} & \left(n_{1}+n_{2}\right)<n
\end{array}\right\}
$$


International Journal of Mathematical, Engineering and Management Sciences

Vol. 6, No. 1, 296-308, 2021

https://doi.org/10.33889/IJMEMS.2021.6.1.019

where,

$$
S_{3}=\sum_{a_{1}=1}^{\min \left(k_{c f}-1-\left(n-n_{1}\right), n_{1}-1\right)} q_{3}^{n-\left(n_{1}+n_{2}\right)} q_{2}{ }^{n_{2}} q_{1}^{a_{1}} \cdot f_{1}\left(i, n_{1}-a_{1}\right)
$$

and

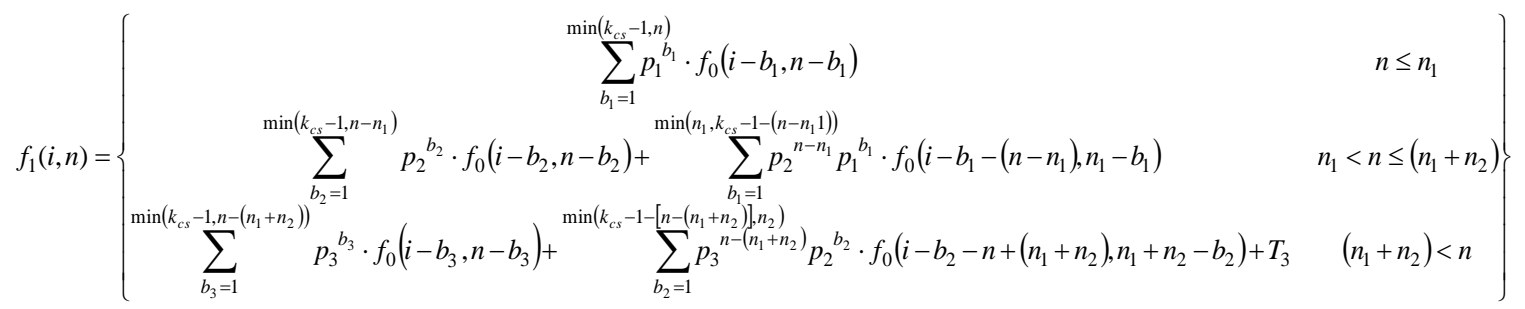

$T_{3}=\sum_{b_{1}=1}^{\min \left(k_{c s}-1-\left(n-n_{1}\right), n_{1}-1\right)} p_{3}{ }^{n-\left(n_{1}+n_{2}\right)} p_{2}{ }^{n_{2}} p_{1}{ }^{b_{1}} \cdot f_{0}\left(i-b_{1}-n+n_{1}, n_{1}-b_{1}\right)$.

Everywhere, $\mathrm{f}_{0}(0,0)=1$.

The boundary conditions are given by:

$$
\begin{aligned}
& f_{0}(0, n)=\left\{\begin{array}{cc}
q_{1}{ }^{n} & n \leq n_{1} \\
q_{1}^{n_{1}} q_{2}{ }^{n-n_{1}} & n_{1}<n \leq n_{1}+n_{2} \\
q_{1}{ }^{n_{1}} q_{2}{ }^{n_{2}} q_{3}{ }^{n-\left(n_{1}+n_{2}\right)} & n_{1}+n_{2}<n
\end{array}\right\} \\
& f_{1}(n, n)=\left\{\begin{array}{cc}
p_{1}{ }^{n} & n \leq n_{1} \\
p_{1}^{n_{1}} p_{2}{ }^{n-n_{1}} & n_{1}<n \leq n_{1}+n_{2} \\
p_{1}^{n_{1}} p_{2}{ }^{{ }_{2}} p_{3}{ }^{n-\left(n_{1}+n_{2}\right)} & n_{1}+n_{2}<n
\end{array}\right\} . \\
& f_{0}(i, 1)=q_{1} \cdot \delta[i], \\
& f_{1}(i, 1)=p_{1} \cdot \delta[i-1] .
\end{aligned}
$$

Example G: Two change points. TFCF. Reliability $R(20)$ for various values of $n_{r}$ and of $p_{r}$ (Table $6)$. 
International Journal of Mathematical, Engineering and Management Sciences

Vol. 6, No. 1, 296-308, 2021

https://doi.org/10.33889/IJMEMS.2021.6.1.019

Table 6. The reliability of a two change points system TFCF.

\begin{tabular}{|c|c|c|c|c|c|c|c|c|}
\hline $\mathrm{k}_{\mathrm{f}}$ & $\mathrm{k}_{\mathrm{cf}}$ & $\mathrm{n}_{1}$ & $\mathrm{n}_{2}$ & $\mathrm{n}_{3}$ & $\mathrm{p}_{1}$ & $\mathrm{p}_{2}$ & $\mathrm{p}_{3}$ & $\mathrm{R}$ \\
\hline- & 3 & 5 & 9 & 6 & 0.88 & 0.92 & 0.95 & 0.9893 \\
\hline 5 & - & 5 & 9 & 6 & 0.88 & 0.92 & 0.95 & 0.9813 \\
\hline 5 & 3 & 5 & 9 & 6 & 0.88 & 0.92 & 0.95 & 0.9744 \\
\hline- & 3 & 5 & 9 & 6 & 0.92 & 0.92 & 0.92 & 0.9915 \\
\hline 5 & - & 5 & 9 & 6 & 0.92 & 0.92 & 0.92 & 0.9817 \\
\hline 5 & 3 & 5 & 9 & 6 & 0.92 & 0.92 & 0.92 & 0.9763 \\
\hline- & 3 & 6 & 8 & 6 & 0.88 & 0.92 & 0.95 & 0.9883 \\
\hline 5 & - & 6 & 8 & 6 & 0.88 & 0.92 & 0.95 & 0.9794 \\
\hline 5 & 3 & 6 & 8 & 6 & 0.88 & 0.92 & 0.95 & 0.9719 \\
\hline- & 4 & 5 & 9 & 6 & 0.88 & 0.92 & 0.95 & 0.9991 \\
\hline 6 & - & 5 & 9 & 6 & 0.88 & 0.92 & 0.95 & 0.9962 \\
\hline 6 & 4 & 5 & 9 & 6 & 0.88 & 0.92 & 0.95 & 0.9955 \\
\hline
\end{tabular}

Example H: Two change points, TSCSTFCF. $\mathrm{P}\{\mathrm{N}>20\}$ for various values of $\mathrm{n}_{\mathrm{r}}$ and of $\mathrm{p}_{\mathrm{r}} . \mathrm{n}_{1}=5$, $\mathrm{n}_{2}=9, \mathrm{n}_{3}=6$ (Table 7).

Table 7. $\mathrm{P}\{\mathrm{N}>20\}$ for a two change points system, TSCSTFCF $\mathrm{n}_{1}=5, \mathrm{n}_{2}=9, \mathrm{n}_{3}=6$.

\begin{tabular}{|c|c|c|c|c|c|c|c|}
\hline $\mathrm{k}_{\mathrm{s}}$ & $\mathrm{k}_{\mathrm{cs}}$ & $\mathrm{k}_{\mathrm{f}}$ & $\mathrm{k}_{\mathrm{cf}}$ & $\mathrm{p}_{1}$ & $\mathrm{p}_{2}$ & $\mathrm{p}_{3}$ & $\mathrm{P}\{\mathrm{N}>20\}$ \\
\hline 18 & - & - & - & 0.92 & 0.92 & 0.92 & 0.2121 \\
\hline 18 & - & - & - & 0.88 & 0.92 & 0.95 & 0.2168 \\
\hline 18 & 15 & 5 & 3 & 0.88 & 0.92 & 0.95 & 0.1851 \\
\hline
\end{tabular}

\section{Several Parallel Tests}

Instead of testing a single unit, it is often preferable to test more units in parallel if we have such spare units at our disposal Gera $(2015,2018)$. The number of units is denoted by $M$. Instead of the previous runs of successes or failures, here we deal with planar squares and rectangles. We thus look for the occurrence of a rectangle $M x k_{c s}$ of successes and/or a rectangle $M x k_{c f}$ of failures. Let $R_{M, n, s}$ be the maximal area of rectangles formed by squares till performing the $n^{\prime}$ th stage of parallel tests and let $R_{M, n, f}$ be the appropriate area for failures. In analogy to before, $T_{n, s}$ presents the total number of successes till the $n^{\prime}$ th stage for all $M$ units and likewise for $T_{n f}$.

Like before, consider a single change point (1).

For any $M \geq m \geq 1,2^{M} \geq j \geq 1$, and any $2^{M} \geq r \geq 0$,

$V(m, j)=\left\{\begin{array}{cc}0 & (2 r+1) u \geq j>2 r u \\ 1 & (2 r+2) u \geq j>(2 r+1) u\end{array}\right\}$

For instance, for $M=2$ units,

$V=\left(\begin{array}{llll}0 & 0 & 1 & 1 \\ 0 & 1 & 0 & 1\end{array}\right)$ 
International Journal of Mathematical, Engineering and Management Sciences

Vol. 6, No. 1, 296-308, 2021

https://doi.org/10.33889/IJMEMS.2021.6.1.019

For any column $j$, we denote the relevant column vector by $\underline{V}_{j}$ and by \|\|$\|$ its $L_{1}$ norm.

We need to add a variable ' $j$ ' to the definition of the previous presented auxiliary functions as follows:

$$
f(i, j, n)=P\left\{T_{n, s}=i, R_{M, n, s}<M k_{c s}, R_{M, n, f}<M k_{c f}, \underline{X}_{n}=\underline{V}_{j}\right\}
$$

The following inter-relations are derived:

$$
i \geq 1, n>1 \text { : }
$$

$$
f(i, 1, n)=\left\{\begin{array}{ll}
q_{1}{ }^{2} \sum_{j^{\prime}=1}^{4} f\left(i, j^{\prime \prime} n-1\right) & n \leq n_{1} \\
q_{2}^{2} \sum_{j^{\prime}=1}^{4} f\left(i, j^{\prime}, n-1\right. & n>n_{1}
\end{array}\right\}
$$

$i>1, n>1$ :

$$
f(i, 2, n)=f(i, 3, n)=\left\{\begin{array}{ll}
q_{1} p_{1} \cdot \sum_{j^{\prime}=1}^{4} f\left(i-1, j^{\prime}, n-1\right) & n \leq n_{1} \\
q_{2} p_{2} \cdot \sum_{j^{\prime}=1}^{4} f\left(i-1, j^{\prime}, n-1\right) & n>n_{1}
\end{array}\right\}
$$

For $n \leq n_{1}$ :

$$
f(i, 4, n)=\sum_{a=1}^{\min \left(k_{c s}-1, n_{1}\right)} p_{1}^{2 a} \cdot \sum_{j^{\prime}=1}^{3} f\left(i-2 a, j^{\prime}, n-a\right)
$$

For $n>n_{1}$ :

$$
\begin{aligned}
f(i, 4, n)= & \sum_{a=1}^{\min \left(n-n_{1}, k_{c s}-1\right)} p_{2}{ }^{M a} \cdot \sum_{j^{\prime}=1}^{3} f\left(i-2 a, j^{\prime}, n-a\right) \\
& +\sum_{b=1}^{\min \left(k_{c s}-1-()\right)} p_{2}{ }^{2\left(n-n_{1}\right)} \cdot p_{1}{ }^{2 b} \cdot \sum_{j^{\prime}=1}^{3} f\left(i-2 b-2\left(n-n_{1}\right), n-b-\left(n-n_{1}\right)\right) \\
& +p_{2}{ }^{2\left(n-n_{1}\right)} \cdot p_{1}{ }^{2 n_{1}} \cdot \delta[i-2 n] \cdot u\left[\left(k_{c s}-1\right)-n\right]
\end{aligned}
$$

Boundary conditions:

$$
\begin{aligned}
& i=0, j>0, n \geq 1: \\
& f(0, j, n)=\left\{\begin{array}{cc}
q_{1}{ }^{M n} \cdot \delta[j-1] & n \leq n_{1} \\
q_{1}{ }^{M n_{1}} \cdot q_{2}{ }^{M\left(n-n_{1}\right)} \cdot \delta[j-1] & n>n_{1}
\end{array}\right\}
\end{aligned}
$$


International Journal of Mathematical, Engineering and Management Sciences

Vol. 6, No. 1, 296-308, 2021

https://doi.org/10.33889/IJMEMS.2021.6.1.019

$i \geq 0, j>0, n=1$ :

$f\left(i=\left\|\underline{V}_{j}\right\|, j, 1\right)=p_{1} \underline{\underline{V}}_{j}\left\|_{q_{1}}{ }^{M-}-\underline{V}_{j}\right\|$

Considering a G: system,

$P\{N>n\}=\sum_{i=0}^{n} \sum_{j=1}^{2 \wedge} f(i, j, n)$

and use (10): $R^{(G)}(n)=1-P\{N>n\}$.

Example: $\mathrm{M}=2, \mathrm{n}_{1}=4, \mathrm{p}_{1}=0.5, \mathrm{p}_{2}=0.25, \mathrm{k}_{\mathrm{cs}}=2$ (Table 8).

Table 8. Reliability for planar CS system with a change point $M=2, \mathrm{n}_{1}=4, \mathrm{p}_{1}=0.5, \mathrm{p}_{2}=0.25, \mathrm{k}_{\mathrm{cs}}=2$.

$$
S_{2}=\left(\begin{array}{l}
S \\
S
\end{array}\right), \bar{S}_{2}=\left(\begin{array}{l}
\bar{S} \\
S
\end{array}\right) .
$$

\begin{tabular}{|l|c|c|c|c|c|c|c|}
\hline $\mathrm{n}$ & 1 & 2 & 3 & 4 & 5 & 6 & 7 \\
\hline $\mathrm{P}\{\mathrm{N}>\mathrm{n}\}$ & 1 & 0.9375 & 0.8906 & 0.8438 & 0.8328 & 0.8302 & 0.8271 \\
\hline $\mathrm{P}\{\mathrm{N}=\mathrm{n}\}$ & 0 & 0.0625 & 0.0469 & 0.011 & 0.0026 & 0.0031 & 0.003 \\
& & $S_{2} S_{2}$ & $\bar{S}_{2} S_{2} S_{2}$ & $1 \bar{S}_{2} S_{2} S_{2}$ & $\bar{S}_{2} S_{2} \bar{S}_{2} S_{2} S_{2}$ & & \\
& & & & & $1 \bar{S}_{2} \bar{S}_{2} S_{2} S_{2}$ & & \\
& & & & & 0.1672 & 0.1698 & 0.1729 \\
\hline $\mathrm{R}^{(\mathrm{G})}(\mathrm{n})$ & 0 & 0.0625 & 0.1094 & 0.1562 & & & \\
\end{tabular}

\section{Conclusion}

The TSCSTFCF procedure for evaluating the reliability of consecutive k-out-of-n systems of components (or tests) has been generalized to include change points within the model. Whereas initially it has been assumed that all components (or tests) have identical reliabilities (or probabilities of success), here we considered systems which are comprised of sets of components (tests) each of which have different reliabilities. The change points indicate the distinction between the parts.

A previously developed method of using some auxiliary functions to evaluate the reliability of a $\mathrm{CF}$ system with change points has been presented. A set of recursive equations is created which are solved numerically. A generalization to TFCF systems has been given. A further extension to start-up demonstration tests involving the TSCSTFCF procedures has been added.

A practical application of the technique may be for instance for a long distance oil pipeline system. Such a line may be divided into several sections according to different environmental conditions. Each part exhibits a different value for its reliability. Likewise, sets of tests may be set up according to the difficulty of passing each test.

It should be added that the components (tests) were assumed to be independent. However, normally we meet systems that involve some dependence between the components. Also, we assumed that the components either work or fail. Actually, we often observe degradation in the 
International Journal of Mathematical, Engineering and Management Sciences

Vol. 6, No. 1, 296-308, 2021

https://doi.org/10.33889/IJMEMS.2021.6.1.019

operation of some components (or partial success in a set of tests). Future work should include both dependent and multi-state components (tests).

The possibility of running several tests in parallel has also been examined. Obviously, this may yield some shortening of the testing procedure. An example has been provided for the case of two parallel tests. Again, the case of dependency and of multi-state outputs may be incorporated into the model within future work.

\section{Conflict of Interest}

The author confirms that there is no conflict of interest to declare for this publication.

\section{Acknowledgements}

The author expresses his sincere thanks to the editor and to the reviewers.

\section{References}

Balakrishnan, N., Koutras, M.V., \& Milienos, F.S. (2014). Start-up demonstration test: models, methods and applications, with some unifications. Applied Stochastic Models in Business and Industry, 30(4), 373-413.

Eryilmaz, S. (2016). Consecutive k-out-of-n lines with a change point. Proceedings of the Institution of Mechanical Engineers, Part O: Journal of Risk and Reliability, 230(6), 545-550.

Gera, A.E. (2010). A new start-up demonstration test. IEEE Transactions on Reliability, 59(1), 128-131.

Gera, A.E. (2011). A general model for start-up demonstration tests. IEEE Transactions on Reliability, 60(1), 295-304.

Gera, A.E. (2015). Start-up demonstration tests involving a two-dimensional TSCSTFCF procedure. International Journal of Reliability, Quality and Safety Engineering, 22(1), 1550003, doi.org/10.1142/s021853931550003.

Gera, A.E. (2018). A comparison of start-up demonstration test procedures based on a combinatorial approach. International Journal of Mathematical, Engineering and Management Sciences, 3(3), 195219.

Gera, A.E. (2019). The TSCSTFCF Procedure for reliability demonstration. Mathematics Applied to Engineering and Management. CRC Press, Boca Raton, 205, DOI:10.1201/9781351123303-8.

Kuo, W., \& Zuo, M.J. (2003). Optimal reliability modeling, principles and applications. John Wiley \& Sons, New York, NY.

Peng, R., \& Xiao, H. (2018). Reliability of linear consecutive k-out-of-n systems with two change points. IEEE Transactions on Reliability, 67(3), 1019-1029, DOI:10.1109/TR.2018.2829709.

Smith, M.L., \& Griffith, W.S. (2011). Multi-state start-up demonstration tests. International Journal of Reliability Quality and Safety Engineering, 18(2), 99-117. 